\title{
Iterative Transceiver Design for MIMO AF Relay Networks with Multiple Sources
}

\author{
Shaodan $\mathrm{Ma}^{\S \dagger}$, Chengwen Xing ${ }^{\dagger}$, Yijia Fan ${ }^{\S}$, Yik-Chung $\mathrm{Wu}^{\dagger}$, Tung-Sang $\mathrm{Ng}^{\dagger}$ and $\mathrm{H}$. Vincent Poor ${ }^{\S}$ \\ $\S$ Department of Electrical Engineering \\ Princeton University \\ Princeton, New Jersey \\ \{shaodanm,yijiafan,poor $\} @$ princeton.edu \\ ${ }^{\dagger}$ Department of Electrical and Electronic Engineering \\ The University of Hong Kong \\ Pokfulam Road, Hong Kong \\ \{sdma,cwxing,ycwu,tsng\}@eee.hku.hk
}

\begin{abstract}
This paper addresses the problem of transceiver design for an amplify-and-forward relay network with multiple sources, multiple relays and multiple destinations. Each node in the network is assumed to be equipped with multiple antennas. A general iterative algorithm is proposed based on convex quadratic optimization theory to minimize mean-square-error of the recovered signals at the destinations. Its convergence and extensions to other scenarios are also discussed. Finally, the effectiveness of the proposed iterative algorithm is demonstrated by computer simulations.
\end{abstract}

\section{INTRODUCTION}

With great potential to enhance coverage and quality of wireless links, cooperative communications has gained considerable attention in recent years [1], [2]. It is generally a multiuser framework which involves three kinds of nodes, i.e., source, relay and destination. The role of the relay is to facilitate communication between source and destination. According to the signal processing performed at the relay, cooperation strategies can be usually classified as amplifyand-forward (AF), decode-and-forward (DF), and compressand-forward $(\mathrm{CF})$. For the AF cooperative strategy, the relay simply forwards the received signal from the source to the destination without knowing the content of the signal. It has lower implementation complexity than the others and thus is preferable for practical applications.

Another important technique introduced in the past decades is multiple input multiple output (MIMO) transmission. With multiple antennas equipped at both transmitter and receiver, spatial diversity and multiplexing gain can be provided without extra bandwidth [3]. This technique has be widely adopted in a host of applications, such as wireless metropolitan area networks (WIMAX) and long term evolution (LTE) systems. Lately, it has also been introduced into cooperative systems and the resulting systems with benefits from MIMO as well as cooperation have attracted considerable research interest [4][13].

Transceiver design, that is, the design of precoder at the transmitter and equalizer at the receiver, is of great importance in MIMO cooperative systems to achieve high-speed reliable communications. A number of studies have been reported in the literature. Most of them however consider simple scenarios with only one source and one destination [5]-[7], [9], [10], [12]. When multiple sources, multiple destinations and multiple relays are involved, the transceiver design becomes very challenging due to the existence of not only multi-antenna interference but also multi-source interference.

In this paper, a dual-hop AF MIMO relay network with multiple relays and multiple pairs of sources and destinations is considered. In such a network, each source transmits multiple data streams through different antennas to its paired destination with the aid of multiple relays. Our aim here is to design the transceiver to maximize the accuracy of the signals recovered at the destinations. Specifically, it is to minimize mean square error (MSE) of the received signals at the destinations. Based on convex quadratic optimization theory, a general iterative algorithm is proposed to jointly design the equalizers at the destinations and the precoders at the relays and sources. Its convergence and extensions to other scenarios are also discussed in detail. Computer simulations finally demonstrate the effectiveness of the proposed algorithm.

The following notation is used throughout this paper. Boldface lowercase letters denote vectors, while boldface uppercase letters denote matrices. The notations $\mathbf{Z}^{\mathrm{T}}, \mathbf{Z}^{\mathrm{H}}$ and $\mathbf{Z}^{*}$ denote the transpose, Hermitian and conjugate of the matrix $\mathbf{Z}$, respectively, and $\operatorname{Tr}(\mathbf{Z})$ represents the trace of the matrix $\mathbf{Z}$. The symbol $\mathbf{I}_{M}$ denotes the $M \times M$ identity matrix, while $\mathbf{0}_{M \times N}$ denotes the $M \times N$ all-zero matrix. The notation $\mathbf{Z}^{\frac{1}{2}}$ is the Hermitian square root of the positive semi-definite matrix $\mathbf{Z}$, such that $\mathbf{Z}=\mathbf{Z}^{\frac{1}{2}} \mathbf{Z}^{\frac{1}{2}}$ and $\mathbf{Z}^{\frac{1}{2}}$ is a Hermitian matrix. The symbol $\mathbb{E}\{\cdot\}$ denotes expectation. The operation vec $(\mathbf{Z})$ stacks the columns of the matrix $\mathbf{Z}$ into a single column vector. The symbol $\otimes$ signifies the Kronecker product.

\section{System Model AND Problem Formulation}

In this paper, a dual-hop AF relay network with $K$ pairs of source and destination nodes and $K_{r}$ relay nodes is considered. As shown in Fig. 1, each node is equipped with multiple antennas and each source would transmit multiple data streams to its paired destination. Let $\mathbf{s}_{k}$ denote the data vector transmitted from the $k^{\text {th }}$ source to its paired destination with covariance matrix $\mathbf{R}_{\mathbf{s}_{k}}=\mathbb{E}\left\{\mathbf{s}_{k} \mathbf{s}_{k}^{\mathrm{H}}\right\}$. Without loss of generality, it is assumed that the signals transmitted from different sources are independent. Before transmission, the data vector $\mathbf{s}_{k}$ is first multiplied with a precoder matrix $\mathbf{W}_{k}$ under a power constraint of the form $\operatorname{Tr}\left(\mathbf{W}_{k} \mathbf{R}_{\mathbf{s}_{k}} \mathbf{W}_{k}^{\mathrm{H}}\right) \leq P_{s, k}$, where $P_{s, k}$ 


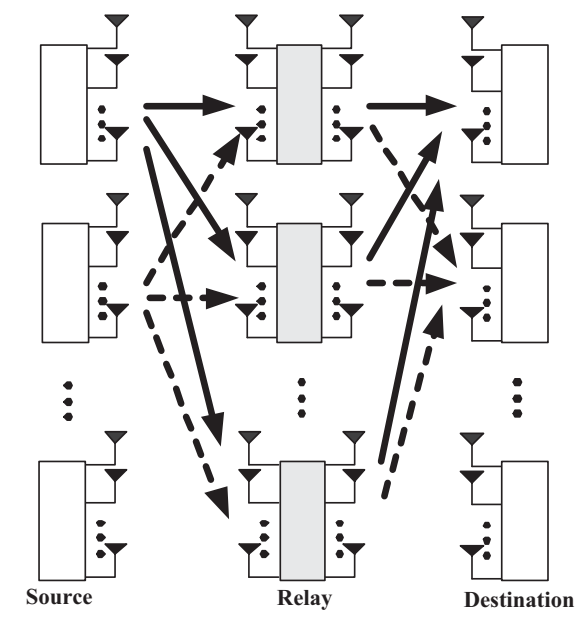

Fig. 1. An AF MIMO relay network.

denotes the maximum transmit power at the $k^{\text {th }}$ source node. On the first hop, all source nodes simultaneously transmit the coded data to the relay nodes. The received signal, $\mathbf{x}_{j}$, at the $j^{\text {th }}$ relay node is

$$
\mathbf{x}_{j}=\mathbf{H}_{s r, i j} \mathbf{W}_{i} \mathbf{s}_{i}+\sum_{k \neq i}\left(\mathbf{H}_{s r, k j} \mathbf{W}_{k} \mathbf{s}_{k}\right)+\mathbf{n}_{1, j}
$$

In (1), $\mathbf{H}_{s r, k j}$ represents the MIMO channel matrix between the $k^{\text {th }}$ source node and the $j^{\text {th }}$ relay node. $\mathbf{n}_{1, j}$ denotes zero-mean additive Gaussian noise with covariance matrix $\mathbf{R}_{\mathbf{n}_{1, j}}$. At the $j^{\text {th }}$ relay node, the received signal $\mathbf{x}_{j}$ is multiplied by a precoder matrix $\mathbf{F}_{j}$, under a power constraint $\operatorname{Tr}\left(\mathbf{F}_{j} \mathbf{R}_{\mathbf{x}_{j}} \mathbf{F}_{j}^{\mathrm{H}}\right) \leq P_{r, j}$, where $\mathbf{R}_{\mathbf{x}_{j}}=\mathbb{E}\left\{\mathbf{x}_{j} \mathbf{x}_{j}^{\mathrm{H}}\right\}$ and $P_{r, j}$ denotes the maximum transmit power at the $j^{\text {th }}$ relay node. Then the resulting signal is transmitted to the destinations on the second hop. The received signal at the $k^{\text {th }}$ destination, $\mathbf{y}_{k}$, can be written as

$$
\begin{aligned}
\mathbf{y}_{k} & =\sum_{j}\left(\mathbf{H}_{r d, j k} \mathbf{F}_{j} \mathbf{x}_{j}\right)+\mathbf{n}_{2, k} \\
& =\sum_{j}\left(\mathbf{H}_{r d, j k} \mathbf{F}_{j} \mathbf{H}_{s r, k j} \mathbf{W}_{k} \mathbf{s}_{k}\right) \\
& +\sum_{j}\left(\mathbf{H}_{r d, j k} \mathbf{F}_{j} \sum_{i \neq k}\left(\mathbf{H}_{s r, i j} \mathbf{W}_{i} \mathbf{s}_{i}\right)\right) \\
& +\sum_{j}\left(\mathbf{H}_{r d, j k} \mathbf{F}_{j} \mathbf{n}_{1, j}\right)+\mathbf{n}_{2, k},
\end{aligned}
$$

where $\mathbf{H}_{r d, j k}$ denotes the MIMO channel matrix between the $j^{\text {th }}$ relay and the $k^{\text {th }}$ destination, and $\mathbf{n}_{2, k}$ denotes a zeromean additive Gaussian noise vector on the second hop with covariance matrix $\mathbf{R}_{\mathbf{n}_{2, k}}$.

At the $k^{\text {th }}$ destination, a linear equalizer $\mathbf{G}_{k}$ is applied to recover the signal transmitted from its paired source, i.e., the $k^{\text {th }}$ source. The MSE of the detected signal at the $k^{\text {th }}$ destination can therefore be formulated as

$$
\begin{aligned}
& \operatorname{MSE}_{k} \\
& =\mathbb{E}\left\{\left\|\mathbf{G}_{k} \mathbf{y}_{k}-\mathbf{s}_{k}\right\|^{2}\right\} \\
& =\operatorname{Tr}\left(\left(\mathbf{G}_{k} \sum_{j}\left(\mathbf{H}_{r d, j k} \mathbf{F}_{j} \mathbf{H}_{s r, k j}\right) \mathbf{W}_{k}-\mathbf{I}\right) \mathbf{R}_{\mathbf{s}_{k}}\right. \\
& \left.\left.\qquad \mathbf{G}_{k} \sum_{j}\left(\mathbf{H}_{r d, j k} \mathbf{F}_{j} \mathbf{H}_{s r, k j}\right) \mathbf{W}_{k}-\mathbf{I}\right)^{\mathrm{H}}\right) \\
& +\operatorname{Tr}\left(\mathbf { G } _ { k } \left(\sum _ { j } \left(\mathbf{H}_{r d, j k} \mathbf{F}_{j} \sum_{i \neq k}\left(\mathbf{H}_{s r, i j} \mathbf{W}_{i} \mathbf{R}_{\mathbf{s}_{i}} \mathbf{W}_{i}^{\mathrm{H}} \mathbf{H}_{s r, i j}^{\mathrm{H}}\right)\right.\right.\right. \\
& \left.\left.\left.\quad \mathbf{F}_{j}^{\mathrm{H}} \mathbf{H}_{r d, j k}^{\mathrm{H}}\right)\right) \mathbf{G}_{k}^{\mathrm{H}}\right)+\operatorname{Tr}\left(\mathbf{G}_{k} \mathbf{R}_{\mathbf{n}_{2, k}} \mathbf{G}_{k}^{\mathrm{H}}\right) \\
& +\operatorname{Tr}\left(\mathbf{G}_{k}\left(\sum_{j}\left(\mathbf{H}_{r d, j k} \mathbf{F}_{j} \mathbf{R}_{\mathbf{n}_{1, j}} \mathbf{F}_{j}^{\mathrm{H}} \mathbf{H}_{r d, j k}^{\mathrm{H}}\right)\right) \mathbf{G}_{k}^{\mathrm{H}}\right) .
\end{aligned}
$$

Obviously, the second term in the MSE is due to the interference from other sources. This term does not exist in single source systems.

Here our objective is to maximize the accuracy of the detected signals at the destinations under transmit power constraints at the sources and relays. It is equivalent to minimize the total MSE of the detected signals. Mathematically, the problem can be formulated as

$$
\begin{array}{ll}
\min _{\mathbf{G}_{k}, \mathbf{F}_{j}, \mathbf{W}_{k}} \quad \overline{\mathrm{MSE}} \triangleq \sum_{k} \mathrm{MSE}_{k} \\
\text { s.t. } & \operatorname{Tr}\left(\mathbf{F}_{j} \mathbf{R}_{\mathbf{x}_{j}} \mathbf{F}_{j}^{\mathrm{H}}\right) \leq P_{r, j}, \quad j=1,2, \ldots, K_{r}, \\
& \operatorname{Tr}\left(\mathbf{W}_{k} \mathbf{R}_{\mathbf{s}_{k}} \mathbf{W}_{k}^{\mathrm{H}}\right) \leq P_{s, k}, \quad k=1,2, \ldots, K .
\end{array}
$$

\section{Proposed Solution}

Generally speaking, transceiver design for cooperative systems with multiple sources, multiple relays and multiple destinations is a very difficult task even for a single antenna configuration, since each relay is shared by multiple sources and multi-source interference exists at both relays and destinations. In the following, an iterative design algorithm is proposed based on convex quadratic optimization theory. Specifically, it iteratively computes the destination equalizers $\mathbf{G}_{k}$, relay precoder matrices $\mathbf{F}_{j}$ and source precoder matrices $\mathbf{W}_{k}$, starting with initial values for $\mathbf{W}_{k}$ and $\mathbf{F}_{j}$. Its convergence and extensions are also discussed in detail.

\section{A. Equalizer Design at Destinations}

Given all the precoder matrices at sources and relays, i.e., $\mathbf{W}_{k}$ and $\mathbf{F}_{j}$, the optimization problem in (4) is an unconstrained convex quadratic optimization problem for each equalizer $\mathbf{G}_{k}$. Then the following condition is necessary and sufficient for the optimal equalizer at the $k^{\text {th }}$ destination:

$$
\frac{\partial \sum_{k} \mathrm{MSE}_{k}}{\partial \mathbf{G}_{k}{ }^{*}}=\mathbf{0} .
$$

Substituting (3) into (5), we have

$$
\begin{aligned}
& \mathbf{G}_{k}\left[\left(\left(\sum_{j} \mathbf{H}_{r d, j k} \mathbf{F}_{j} \mathbf{H}_{s r, k j}\right) \mathbf{W}_{k}\right) \mathbf{R}_{\mathbf{s}_{k}}\left(\left(\sum_{j} \mathbf{H}_{r d, j k} \mathbf{F}_{j} \mathbf{H}_{s r, k j}\right) \mathbf{W}_{k}\right)^{\mathrm{H}}\right. \\
& \left.+\mathbf{R}_{\mathbf{n}_{k}}\right]=\mathbf{R}_{\mathbf{s}_{k}}\left(\left(\sum_{j} \mathbf{H}_{r d, j k} \mathbf{F}_{j} \mathbf{H}_{s r, k j}\right) \mathbf{W}_{k}\right)^{\mathrm{H}}
\end{aligned}
$$


in which the matrix $\mathbf{R}_{\mathbf{n}_{k}}$ is defined as

$$
\begin{aligned}
& \mathbf{R}_{\mathbf{n}_{k}} \triangleq \sum_{j}\left(\mathbf{H}_{r d, j k} \mathbf{F}_{j} \mathbf{R}_{\mathbf{n}_{1, j}} \mathbf{F}_{j}^{\mathrm{H}} \mathbf{H}_{r d, j k}^{\mathrm{H}}\right) \\
& +\sum_{j}\left(\mathbf{H}_{r d, j k} \mathbf{F}_{j} \sum_{i \neq k}\left(\mathbf{H}_{s r, i j} \mathbf{W}_{i} \mathbf{R}_{\mathbf{s}_{i}} \mathbf{W}_{i}^{\mathrm{H}} \mathbf{H}_{s r, i j}^{\mathrm{H}}\right)\right. \\
& \left.\mathbf{F}_{j}^{\mathrm{H}} \mathbf{H}_{r d, j k}^{\mathrm{H}}\right)+\mathbf{R}_{\mathbf{n}_{2, k}} .
\end{aligned}
$$

It follows that the optimal equalizer at the $k^{\text {th }}$ destination is given by

$$
\begin{aligned}
& \mathbf{G}_{k}=\mathbf{R}_{\mathbf{s}_{k}}\left(\left(\sum_{j} \mathbf{H}_{r d, j k} \mathbf{F}_{j} \mathbf{H}_{s r, k j}\right) \mathbf{W}_{k}\right)^{\mathrm{H}} \\
& {\left[\left(\left(\sum_{j} \mathbf{H}_{r d, j k} \mathbf{F}_{j} \mathbf{H}_{s r, k j}\right) \mathbf{W}_{k}\right) \mathbf{R}_{\mathbf{s}_{k}}\left(\left(\sum_{j} \mathbf{H}_{r d, j k} \mathbf{F}_{j} \mathbf{H}_{s r, k j}\right) \mathbf{W}_{k}\right)^{\mathrm{H}}\right.} \\
& \left.+\mathbf{R}_{\mathbf{n}_{k}}\right]^{-1} .
\end{aligned}
$$

Notice that to the best of our knowledge, for almost all the estimation problems based the MMSE criterion, the equalizer/estimator design problem can be formulated as an unconstrained convex quadratic optimization problem [14]. The optimal solution is thereby easy to achieve based on the first derivative of the objective function.

\section{B. Precoder Matrix Design at Relays}

When the destination equalizers $\mathbf{G}_{k}$ and the source precoder matrices $\mathbf{W}_{k}$ are fixed, the optimization problem in (4) is a convex quadratic optimization problem for the precoder matrix $\mathbf{F}_{j}$ at the $j^{\text {th }}$ relay with only one transmit power constraint. For this constrained problem, the Karush-Kuhn-Tucker (KKT) conditions [15] which are necessary and sufficient conditions for an optimal solution, are given as follows:

$$
\begin{aligned}
& \sum_{k}\left[\mathbf{H}_{r d, j k}^{\mathrm{H}} \mathbf{G}_{k}^{\mathrm{H}} \mathbf{G}_{k}\left(\sum_{i \neq j}\left(\mathbf{H}_{r d, i k} \mathbf{F}_{i} \mathbf{H}_{s r, k i}\right)\right) \mathbf{W}_{k} \mathbf{R}_{\mathbf{s}_{k}} \mathbf{W}_{k}^{\mathrm{H}}\right. \\
& \left.\mathbf{H}_{s r, k j}^{\mathrm{H}}\right]+\lambda_{j} \mathbf{F}_{j} \mathbf{R}_{\mathbf{x}_{j}}+\sum_{k}\left(\mathbf{H}_{r d, j k}^{\mathrm{H}} \mathbf{G}_{k}^{\mathrm{H}} \mathbf{G}_{k} \mathbf{H}_{r d, j k}\right) \\
& \mathbf{F}_{j} \sum_{l}\left(\mathbf{H}_{s r, l j}\left(\mathbf{W}_{l} \mathbf{R}_{\mathbf{s}_{l}} \mathbf{W}_{l}^{\mathrm{H}}\right) \mathbf{H}_{s r, l j}^{\mathrm{H}}\right) \\
& +\sum_{k}\left(\mathbf{H}_{r d, j k}^{\mathrm{H}} \mathbf{G}_{k}^{\mathrm{H}} \mathbf{G}_{k} \mathbf{H}_{r d, j k}\right) \mathbf{F}_{j} \mathbf{R}_{\mathbf{n}_{1, j}} \\
& =\sum_{k}\left(\mathbf{H}_{r d, j k}^{\mathrm{H}} \mathbf{G}_{k}^{\mathrm{H}} \mathbf{W}_{k}^{\mathrm{H}} \mathbf{R}_{\mathbf{s}_{k}} \mathbf{H}_{s r, k j}^{\mathrm{H}}\right) \\
& \lambda_{j}\left(\operatorname{Tr}\left(\mathbf{F}_{j} \mathbf{R}_{\mathbf{x}_{j}} \mathbf{F}_{j}^{\mathrm{H}}\right)-P_{r, j}\right)=0 \\
& \operatorname{Tr}\left(\mathbf{F}_{j} \mathbf{R}_{\mathbf{x}_{j}} \mathbf{F}_{j}^{\mathrm{H}}\right) \leq P_{r, j} \\
& \lambda_{j}(0 .
\end{aligned}
$$

and $\lambda_{j} \geq 0$.

Using the fact that the covariance matrix of the received signal $\mathbf{x}_{j}$ at the $j^{\text {th }}$ relay is

$$
\mathbf{R}_{\mathbf{x}_{j}}=\sum_{k}\left(\mathbf{H}_{s r, k j} \mathbf{W}_{k} \mathbf{R}_{\mathbf{s}_{k}} \mathbf{W}_{k}^{\mathrm{H}} \mathbf{H}_{s r, k j}^{\mathrm{H}}\right)+\mathbf{R}_{\mathbf{n}_{1, j}}
$$

the first KKT condition in (9) can be rewritten as

$$
\begin{aligned}
& \sum_{k}\left[\mathbf{H}_{r d, j k}^{\mathrm{H}} \mathbf{G}_{k}^{\mathrm{H}} \mathbf{G}_{k}\left(\sum_{i \neq j}\left(\mathbf{H}_{r d, i k} \mathbf{F}_{i} \mathbf{H}_{s r, k i}\right)\right) \mathbf{W}_{k} \mathbf{R}_{\mathbf{s}_{k}} \mathbf{W}_{k}^{\mathrm{H}}\right. \\
& \left.\mathbf{H}_{s r, k j}^{\mathrm{H}}\right]+\lambda_{j} \mathbf{F}_{j} \mathbf{R}_{\mathbf{x}_{j}}+\sum_{k}\left(\mathbf{H}_{r d, j k}^{\mathrm{H}} \mathbf{G}_{k}^{\mathrm{H}} \mathbf{G}_{k} \mathbf{H}_{r d, j k}\right) \mathbf{F}_{j} \mathbf{R}_{\mathbf{x}_{j}} \\
& =\sum_{k}\left(\mathbf{H}_{r d, j k}^{\mathrm{H}} \mathbf{G}_{k}^{\mathrm{H}} \mathbf{W}_{k}^{\mathrm{H}} \mathbf{R}_{\mathbf{s}_{k}} \mathbf{H}_{s r, k j}^{\mathrm{H}}\right),
\end{aligned}
$$

based on which the optimal precoding matrix at the $j^{\text {th }}$ relay is derived as

$$
\begin{aligned}
& \mathbf{F}_{j}=\left(\lambda_{j} \mathbf{I}+\sum_{k}\left(\mathbf{H}_{r d, j k}^{\mathrm{H}} \mathbf{G}_{k}^{\mathrm{H}} \mathbf{G}_{k} \mathbf{H}_{r d, j k}\right)\right)^{-1} \\
& \sum_{k}\left[\mathbf { H } _ { r d , j k } ^ { \mathrm { H } } \mathbf { G } _ { k } ^ { \mathrm { H } } \left(\mathbf{I}-\mathbf{G}_{k}\left(\sum_{i \neq j}\left(\mathbf{H}_{r d, i k} \mathbf{F}_{i} \mathbf{H}_{s r, k i}\right) \mathbf{W}_{k}\right)\right.\right. \\
& \left.\mathbf{W}_{k}^{\mathrm{H}} \mathbf{R}_{\mathbf{s}_{k}} \mathbf{H}_{s r, k j}^{\mathrm{H}}\right] \mathbf{R}_{\mathbf{x}_{j}}^{-1} .
\end{aligned}
$$

Obviously from (15), in order to compute the optimal $\mathbf{F}_{j}$, the Lagrange multiplier $\lambda_{j}$ should be calculated first. However, there is no closed-form solution for $\lambda_{j}$ simultaneously satisfying (10) and (11). Below we propose a low complexity method to solve (10) and (11).

First, notice that in order to have (10) satisfied, either $\lambda_{j}=0$ or $\operatorname{Tr}\left(\mathbf{F}_{j} \mathbf{R}_{\mathbf{x}_{j}} \mathbf{F}_{j}^{\mathrm{H}}\right)=P_{r, j}$ must hold. If $\lambda_{j}=0$ also makes (11) satisfied, $\lambda_{j}=0$ is a solution to (10) and (11). Since given $\mathbf{G}_{k}$ and $\mathbf{W}_{k}$, the optimization problem (4) is a convex quadratic problem in $\mathbf{F}_{j}$. It has only one solution for $\mathbf{F}_{j}$ and thus $\lambda_{j}=0$ is the only solution to (10) and (11) in this case. On other hand, if $\lambda_{j}=0$ does not make (11) satisfied, we have to solve $\operatorname{Tr}\left(\mathbf{F}_{j} \mathbf{R}_{\mathbf{x}_{j}} \mathbf{F}_{j}^{\mathrm{H}}\right)=P_{r, j}$. It can be proven that when $\mathbf{G}_{k}$ and $\mathbf{W}_{k}$ are fixed, the function $f_{j}\left(\lambda_{j}\right)=\operatorname{Tr}\left(\mathbf{F}_{j} \mathbf{R}_{\mathbf{x}_{j}} \mathbf{F}_{j}^{\mathrm{H}}\right)$ is a decreasing function of $\lambda_{j}$ which satisfies

$$
0 \leq \lambda_{j} \leq \sqrt{\frac{\operatorname{Tr}\left(\mathbf{M}_{j} \mathbf{R}_{\mathbf{x}_{j}} \mathbf{M}_{j}^{\mathrm{H}}\right)}{P_{r, j}}},
$$

where $\mathbf{M}_{j}$ is defined as

$$
\begin{aligned}
\mathbf{M}_{j} \triangleq & \sum_{k}\left[\mathbf { H } _ { r d , j k } ^ { \mathrm { H } } \mathbf { G } _ { k } ^ { \mathrm { H } } \left(\mathbf{I}-\mathbf{G}_{k}\left(\sum_{i \neq j}\left(\mathbf{H}_{r d, i k} \mathbf{F}_{i} \mathbf{H}_{s r, k i}\right) \mathbf{W}_{k}\right)\right.\right. \\
& \left.\mathbf{W}_{k}^{\mathrm{H}} \mathbf{R}_{\mathbf{s}_{k}} \mathbf{H}_{s r, k j}^{\mathrm{H}}\right] \mathbf{R}_{\mathbf{x}_{j}}^{-1} .
\end{aligned}
$$

Due to space limitations, the proof is not presented here. Based on this result, $\lambda_{j}$ can be efficiently computed by a onedimension search, such as bisection or golden search [17]. Since $\operatorname{Tr}\left(\mathbf{F}_{j} \mathbf{R}_{\mathbf{x}_{\mathbf{j}}} \mathbf{F}_{j}^{\mathrm{H}}\right)=P_{r, j}$ is a stronger condition than $\operatorname{Tr}\left(\mathbf{F}_{j} \mathbf{R}_{\mathbf{x}_{\mathbf{j}}} \mathbf{F}_{j}^{\mathrm{H}}\right) \leq P_{r, j}$, (11) is satisfied automatically in this case. In summary, we take $\lambda_{j}=0$, if $f_{j}(0) \leq P_{r, j}$, and solve $f_{j}\left(\lambda_{j}\right)=P_{r, j}$ otherwise.

Notice that given the destination equalizers $\mathbf{G}_{k}$ and the source precoder matrices $\mathbf{W}_{k}$, the optimization problem for the precoder matrix $\mathbf{F}_{j}$ at the $j^{\text {th }}$ relay can also be transformed into a semi-definite programming (SDP) problem which can 
be efficiently solved by interior point polynomial algorithms. However, this algorithm shows higher complexity than the algorithm we propose here.

\section{Precoder Matrix Design at Sources}

From the constraint formulation in (4) and the definition of $\mathbf{R}_{\mathbf{x}_{j}}$ in (13), we notice that every source precoder matrix is involved in power constraints at one source and all $K_{r}$ relays. Given the destination equalizers $\mathbf{G}_{k}$ and relay precoder matrices $\mathbf{F}_{j}$, the optimization problem (4) is therefore a convex quadratic optimization problem for the precoder matrix $\mathbf{W}_{k}$ at the $k^{\text {th }}$ source with $K_{r}+1$ constraints. For this optimization problem, it is difficult to compute the optimal solution directly based on KKT conditions, since multiple Lagrange multipliers, each corresponding to one constraint, need to be solved. However, based on (3), the problem can be reformulated as the following quadratic optimization problem:

$$
\begin{array}{cl}
\min _{\mathbf{W}_{k}} & \operatorname{Tr}\left(\overline{\mathbf{N}}^{\mathrm{H}} \mathbf{W}_{k}^{\mathrm{H}} \overline{\mathbf{A}} \mathbf{W}_{k} \overline{\mathbf{N}}\right)+2 \mathcal{R}\left\{\operatorname{Tr}\left(\overline{\mathbf{B}}^{\mathrm{H}} \mathbf{W}_{k}\right)\right\}+\bar{c} \\
\text { s.t. } & \operatorname{Tr}\left(\mathbf{N}_{j}^{\mathrm{H}} \mathbf{W}_{k}^{\mathrm{H}} \mathbf{A}_{j} \mathbf{W}_{k} \mathbf{N}_{j}\right)+2 \mathcal{R} \operatorname{Tr}\left\{\left(\mathbf{B}_{j}^{\mathrm{H}} \mathbf{W}_{k}\right)\right\}+c_{j} \leq 0 \\
& j=0,1, \ldots, K_{r},
\end{array}
$$

where $\bar{c}$ is a constant irrelevant to $\mathbf{W}_{k}$ and the other parameters are specified as follows:

$$
\begin{gathered}
\overline{\mathbf{A}}=\sum_{k}\left[\left(\mathbf{G}_{k}\left(\sum_{j} \mathbf{H}_{r d, j k} \mathbf{F}_{j} \mathbf{H}_{s r, k j}\right)\right)^{\mathrm{H}}\right. \\
\left.\left(\mathbf{G}_{k}\left(\sum_{j} \mathbf{H}_{r d, j k} \mathbf{F}_{j} \mathbf{H}_{s r, k j}\right)\right)\right], \\
\overline{\mathbf{B}}=-\left(\mathbf{G}_{k} \sum_{j}\left(\mathbf{H}_{r d, j k} \mathbf{F}_{j} \mathbf{H}_{s r, k j}\right)\right)^{\mathrm{H}}, \quad \overline{\mathbf{N}}=\mathbf{R}_{\mathbf{s}_{k}}^{\frac{1}{2}}, \\
\mathbf{A}_{0}=\mathbf{I}, \quad \mathbf{A}_{j>0}=\mathbf{H}_{s r, k j}^{\mathrm{H}} \mathbf{F}_{j}^{\mathrm{H}} \mathbf{F}_{j} \mathbf{H}_{s r, k j}, \\
\mathbf{B}_{j}=\mathbf{0}, \quad \mathbf{N}_{j}=\mathbf{R}_{\mathbf{s}_{k}}^{\frac{1}{2}}, \quad c_{0}=-P_{s, k} \\
\text { and } \mathbf{c}_{j>0}=\operatorname{Tr}\left(\sum_{l \neq k}\left(\mathbf{F}_{j} \mathbf{H}_{s r, l j} \mathbf{W}_{l} \mathbf{R}_{\mathbf{s}_{l}} \mathbf{W}_{l}^{\mathrm{H}} \mathbf{H}_{s r, l j}^{\mathrm{H}} \mathbf{F}_{j}^{\mathrm{H}}\right)\right) \\
+\operatorname{Tr}\left(\mathbf{F}_{j} \mathbf{R}_{\mathbf{n}_{1, j}} \mathbf{F}_{j}^{\mathrm{H}}\right)-P_{r, j} .
\end{gathered}
$$

Notice that the constraint with $j=0$ in (18) corresponds to the power constraint at the $k^{\text {th }}$ source, while those constraints with $j>0$ correspond to the power constraints at the relays.

Using properties of Kronecker product and the identity $\operatorname{Tr}(\mathbf{A B})=\operatorname{vec}^{\mathrm{H}}\left(\mathbf{A}^{\mathrm{H}}\right) \operatorname{vec}(\mathbf{B})$, the constraints in (18) can be rewritten as

$$
\begin{aligned}
& \operatorname{Tr}\left(\mathbf{N}_{j}^{\mathrm{H}} \mathbf{W}_{k}^{\mathrm{H}} \mathbf{A}_{j} \mathbf{W}_{k} \mathbf{N}_{j}\right)+2 \mathcal{R}\left\{\operatorname{Tr}\left(\mathbf{B}_{j}^{\mathrm{H}} \mathbf{W}_{k}\right)\right\}+c_{j} \\
& =\operatorname{vec}^{\mathrm{H}}\left(\mathbf{A}_{j}^{\frac{1}{2}} \mathbf{W}_{k} \mathbf{N}_{j}\right) \operatorname{vec}\left(\mathbf{A}_{j}^{\frac{1}{2}} \mathbf{W}_{k} \mathbf{N}_{j}\right)+2 \mathcal{R}\left\{\operatorname{vec}^{\mathrm{H}}\left(\mathbf{B}_{j}\right) \operatorname{vec}\left(\mathbf{W}_{k}\right)\right\} \\
& +c_{j} \\
& =\operatorname{vec}^{\mathrm{H}}\left(\mathbf{W}_{k}\right)\left(\mathbf{N}_{j}^{*} \otimes \mathbf{A}_{j}^{\frac{\mathrm{H}}{2}}\right)\left(\mathbf{N}_{j}^{\mathrm{T}} \otimes \mathbf{A}_{j}^{\frac{1}{2}}\right) \operatorname{vec}\left(\mathbf{W}_{k}\right) \\
& +2 \mathcal{R}\left\{\operatorname{vec}^{\mathrm{H}}\left(\mathbf{B}_{j}\right) \operatorname{vec}\left(\mathbf{W}_{k}\right)\right\}+c_{j} \leq 0
\end{aligned}
$$

based on which and together with Schur complement lemma [15], the optimization problem in (18) can be reformulated as the following SDP problem:

$$
\begin{aligned}
& \min _{\mathbf{W}_{k}} t \\
& \text { s.t. } \\
& {\left[\begin{array}{cc}
\mathbf{I} & \left(\overline{\mathbf{N}}^{\mathrm{T}} \otimes \overline{\mathbf{A}}^{\frac{1}{2}}\right) \operatorname{vec}\left(\mathbf{W}_{k}\right) \\
\left(\left(\overline{\mathbf{N}}^{\mathrm{T}} \otimes \overline{\mathbf{A}}^{\frac{1}{2}}\right) \operatorname{vec}\left(\mathbf{W}_{k}\right)\right)^{\mathrm{H}} & -2 \mathcal{R}\left(\operatorname{vec}^{\mathrm{H}}(\overline{\mathbf{B}}) \operatorname{vec}\left(\mathbf{W}_{k}\right)\right)+t
\end{array}\right]} \\
& \succeq 0 \\
& \left.\begin{array}{cc}
\succeq & \left(\mathbf{N}_{j}^{\mathrm{T}} \otimes \mathbf{A}_{j}^{\frac{1}{2}}\right) \operatorname{vec}\left(\mathbf{W}_{k}\right) \\
\left(\left(\mathbf{N}_{j}^{\mathrm{T}} \otimes \mathbf{A}_{j}^{\frac{1}{2}}\right) \operatorname{vec}\left(\mathbf{W}_{k}\right)\right)^{\mathrm{H}} & -2 \mathcal{R}\left(\operatorname{vec}^{\mathrm{H}}\left(\mathbf{B}_{j}\right) \operatorname{vec}\left(\mathbf{W}_{k}\right)\right)-c_{j}
\end{array}\right] \\
& \succeq 0, \quad j=0,1, \ldots, K_{r} .
\end{aligned}
$$

Then the optimal precoder matrix $\mathbf{W}_{k}$ can be found by solving this SDP problem using numerical tools such as CVX. Notice that in our work, the variables are complex matrices. For some optimization tool boxes, only real variables are permitted. In that case, a minor transformation is needed, which is

$$
\left[\begin{array}{ll}
\mathbf{I}_{N} & \mathbf{v} \\
\mathbf{v}^{\mathrm{H}} & a
\end{array}\right] \succeq 0 \rightarrow\left[\begin{array}{cc}
\mathbf{I}_{2 N} & \tilde{\mathbf{v}} \\
\tilde{\mathbf{v}}^{\mathrm{T}} & a
\end{array}\right] \succeq 0
$$

where

$$
\tilde{\mathbf{v}}=\left[\operatorname{Real}(\mathbf{v})^{\mathrm{T}} \operatorname{Imag}(\mathbf{v})^{\mathrm{T}}\right]^{\mathrm{T}} .
$$

\section{Initialization and Convergence Analysis}

In summary, the proposed algorithm iteratively computes the destination equalizers, relay precoder matrices and source precoder matrices based on (8), (15) and (25), respectively, until $\left\|\overline{\mathrm{MSE}}_{i}-\overline{\mathrm{MSE}}_{i-1}\right\| \leq \theta$ where $\overline{\mathrm{MSE}}_{i}$ is the total MSE at the $i$ th iteration and $\theta$ is a predetermined threshold. At the beginning of the algorithm, initial values for $\mathbf{W}_{k}$ and $\mathbf{F}_{j}$ are needed. Generally, they are initialized to satisfy $\operatorname{Tr}\left(\mathbf{W}_{k} \mathbf{R}_{\mathbf{s}_{k}} \mathbf{W}_{k}^{\mathrm{H}}\right)=P_{s, k}$ and $\operatorname{Tr}\left(\mathbf{F}_{j} \mathbf{R}_{\mathbf{x}_{j}} \mathbf{F}_{j}^{\mathrm{H}}\right)=P_{r, j}$, respectively. For simplicity, they can be initialized as $\mathbf{W}_{k} \propto \mathbf{I}$ and $\mathbf{F}_{j} \propto \mathbf{I}$.

At each step in the iterative algorithm, the optimization problem (4) turns out to be a convex problem for one variable provided that other variables are given. Thus a unique optimal solution leading to a smaller total MSE compared to that in the previous step can always be found for that variable. Consequently, the total MSE is monotonically decreased at each iteration, which proves the convergence of the proposed iterative algorithm. However, only local optimality can be guaranteed by the proposed algorithm, which is a common weakness for most kinds of iterative algorithms [16]. Notice that the optimality of the final solution depends heavily on initial values. When the initial values are close enough to the globally optimal solution, the final solution tends to be globally optimal.

\section{E. Extensions}

The proposed iterative algorithm is a general algorithm to tackle the problem of multiple variable design. It is also applicable to the following scenarios with minor modifications:

1) Weighted MSE, which is more general than the total sum MSE, is considered as an objective function. The weighting 
factors can be chosen according to different criteria to achieve fairness among sources.

2) Direct links between sources and destinations are considered and certain linear schemes are adopted to combine the signals from relay links and source links at the destinations.

3) One source transmits multiple data streams to multiple destinations with the aid of multiple relays. This corresponds to the downlink of a cellular system.

4) Multiple sources transmit data simultaneously to one destination with the aid of multiple relays, which corresponds to the uplink of a cellular system.

\section{Simulation Results and Discussion}

Computer simulations have been conducted to investigate the performance of the proposed algorithm. A network with two pairs of source and destination nodes and one relay node is taken as an example for these simulations. Each source/destination node has two antennas, while the relay node which is shared by two sources has four antennas. Thus, this is a relay network with both multi-source and multi-antenna interference.

In the simulations, the noise variance matrices are set as $\mathbf{R}_{\mathbf{n}_{1,1}}=\sigma_{1}^{2} \mathbf{I}_{4}$ and $\mathbf{R}_{\mathbf{n}_{2,1}}=\mathbf{R}_{\mathbf{n}_{2,2}}=\sigma_{2}^{2} \mathbf{I}_{2}$. The signal-tonoise ratios (SNRs) for the source-relay links are defined as $\mathrm{E}_{s r, k}=P_{s, k} /\left(2 \sigma_{1}^{2}\right), k=1,2$, and are fixed as $\mathrm{E}_{s r, k}=20 \mathrm{~dB}$. At each source node, two independent data streams, each with $N_{\text {Data }}=1000$ independent quadrature phase-shift keying (QPSK) symbols, are transmitted. The SNR for each relaydestination link is defined as $\mathrm{E}_{r d}=P_{r} /\left(4 \sigma_{2}^{2}\right)$. The results shown in the following figure are an average over 200 independent channel realizations.

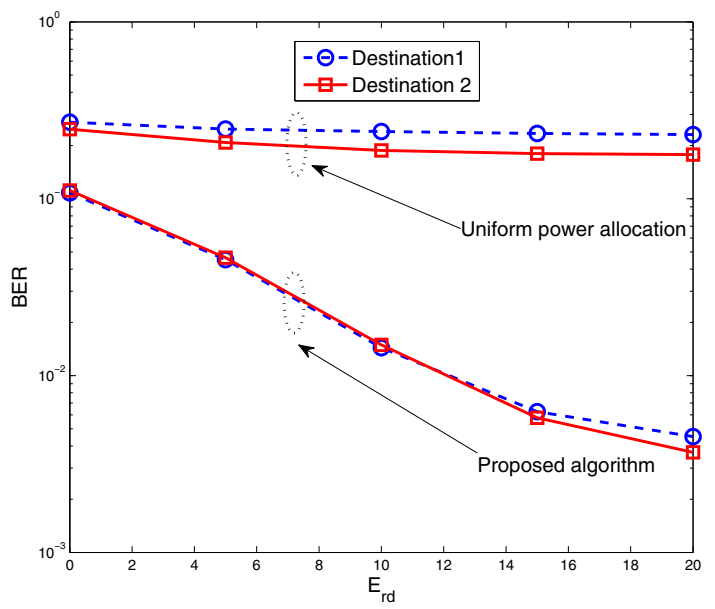

Fig. 2. BER of the proposed algorithm and the uniform power allocation algorithm.

For the purpose of comparison, a uniform power allocation algorithm is also simulated. In this algorithm, the precoder matrices at the sources and relay are proportional to the identity matrix and are designed to satisfy the power constraints with equality, while a linear MMSE equalizer is adopted at each destination node to recover its corresponding signal.
Fig. 2 shows bit-error-rate (BER) performance of the proposed algorithm and the uniform power allocation algorithm. It can be seen that with the precoder matrices proportional to the identity matrix in the uniform power allocation algorithm, increasing the transmit power at the relay cannot improve the system performance. However, through proper design of the precoder matrices and equalizers, the proposed algorithm significantly outperforms the uniform power allocation algorithm and its performance is improved when the relay-destination SNR increases. Notice that since the SNRs for the sourcerelay links are fixed as $20 \mathrm{~dB}$, error floors are shown for both the proposed and uniform power allocation algorithms in the figure.

\section{CONCLUSIONS}

Linear MMSE transceiver design for dual-hop MIMO relay networks has been investigated in this paper. The network considered here is a general one which involves multiple sources, multiple relays and multiple destinations. Using convex quadratic optimization theory, an effective iterative algorithm which guarantees to converge has been proposed. It can be easily extended to several important scenarios, such as the uplink/downlink of a MIMO cellular cooperative system.

\section{REFERENCES}

[1] A. Scaglione, D. L. Goeckel and J. N. Laneman, "Cooperative communications in mobile ad hoc networks," IEEE Signal Processing Magazine, vol. 23, no. 5, pp. 18-29, Sept. 2006.

[2] J. N. Laneman, D. N. C. Tse and G. W. Wornell, "Cooperative diversity in wireless networks: Efficient protocols and outage behavior,' IEEE Trans. on Information Theory, vol. 50, no. 12 pp. 3062-3080, Dec. 2004.

[3] I. E. Telatar, "Capacity of multi-antenna Gaussian channels," European Trans. on Telecommunications, vol. 10, no. 6, pp. 585-595, Nov./Dec. 1999.

[4] B. Wang, J. Zhang and A. Host-Madsen, "On the capacity of MIMO relay channels," IEEE Trans. on Information Theory, vol. 51, no. 1, pp. 29-43, Jan. 2005.

[5] O. Munoz-Medina, J. Vidal and A. Agustin, "Linear transceiver design in nonregenerative relays with channel state information," IEEE Trans. on Signal Processing, vol. 55, no. 6, pp. 2593-2604, June 2007.

[6] X. Tang and Y. Hua, "Optimal design of non-regenerative MIMO wireless relays," IEEE Trans. on Wireless Communications, vol. 6, no. 4, pp. 1398-1407, Apr. 2007.

[7] Y. Fan and J. Thompson, "MIMO configurations for relay channels: Theory and practice," IEEE Trans. on Wireless Communications, vol. 6, no. 5, pp. 1774-1786, May 2007.

[8] C.-B. Chae, T. W. Tang, R. W. Health and S.-Y. Cho, "MIMO relaying with linear processing for multiuser transmission in fixed relay netwrks," IEEE Trans. on Signal Processing, vol. 56, no. 2, pp. 727-738, Feb. 2008.

[9] H. W. Je, B. Lee, S. Kim and K. B. Lee, "Design of non-regenerative MIMO-relay systems with partial channel state information," Proc. of IEEE International Conference on Communications (2008), pp. 44414445, May 2008.

[10] A. S. Behbahani, R. Merched and A. M. Eltawil, "Optimizations of a MIMO relay network, " IEEE Trans. on Signal Processing, vol. 56, no. 10, part 2, pp. 5062-5073, Oct. 2008.

[11] H. Bölcskei, R. U. Nabar, O. Oyman and A. J. Paulraj, "Capacity scaling laws in MIMO relay networks," IEEE Trans. on Wireless Communications, vol. 5, no. 6, pp. 1433-1444, June 2006.

[12] W. Guan and H. W. Luo, "Joint MMSE transceiver design in nonregenerative MIMO relay systems," IEEE Communications Letters, vol. 12, no. 7, pp. 517-519, July 2008.

[13] Y. Yu and Y. Hua, "Power allocation for a MIMO relay system with multiple-antenna users," IEEE Trans. on Signal Processing, vol. 58, no. 5, pp. 2823-2835, May 2010 
[14] S. M. Kay, Fundamentals of Statistical Signal Processing: Estimation Theory. Englewood Cliffs, NJ: Prentice-Hall, 1993.

[15] S. Boyd and L. Vandenberghe, Convex Optimization. Cambridge, UK: Cambridge University Press, 2004.

[16] J. Zhang, Y. Wu, S. Zhou and J. Wang, "Joint linear transmitter and receiver design for the downlink of multiuser MIMO," IEEE Communications Letters, vol. 9, no. 11, pp. 991-993, Nov. 2005.

[17] D. P. Bersekas, Nonlinear Programming, 2nd ed., New York: Athena Scientific, 2004 